\title{
PROCESSO DE BUSCA DE FAMÍLIAS PARA A COLETA DE DADOS EM PESQUISA QUALITATIVA
}

\author{
Astrid Eggert Boehs ${ }^{1}$, Carlos Gabriel Eggert Boehs ${ }^{2}$, Gisele Cristina Manfrini Fernandes ${ }^{3}$, Eliane Regina Pereira \\ do Nascimento ${ }^{4}$
}

${ }^{1}$ Doutora em Enfermagem. Departamento de Enfermagem da Universidade Federal de Santa Catarina (UFSC). Florianópolis, Santa Catarina, Brasil. E-mail: astridboehs@hotmail.com

${ }^{2}$ Doutor em Administração. Empresa Paranaense de Energia. Curitiba, Paraná, Brasil. E-mail: cgeboehs@gmail.com

${ }^{3}$ Doutora em Enfermagem. Docente do Departamento de Enfermagem da UFSC. Florianópolis, Santa Catarina, Brasil. E-mail: gisele.manfrini@ufsc.br

${ }^{4}$ Doutora em Enfermagem. Docente Departamento de Enfermagem e do Programa de Pós Graduação de Enfermagem UFSC. Florianópolis, Santa Catarina, Brasil. E-mail: eliane.nascimento@ufsc.br

\section{RESUMO}

Objetivo: refletir o processo de busca de famílias para pesquisa na área rural.

Método: reflexão sobre o processo de busca realizado em duas pesquisas (A e B) a partir de quatro fatores: o tema, os critérios de seleção, o papel do mediador e a posição do pesquisador em relação ao gênero, idade, profissão e filiação.

Resultados: a análise destes quatro fatores no processo de busca dos participantes mostra que o auxilio dos mediadores foi fundamental para selecionar e acessar as famílias em seus domicílios. Houve dificuldade em ouvir diferentes membros das famílias. Na pesquisa A, houve maior participação dos homens adultos, e na pesquisa B, das mulheres adultas, o que coincidiu com o gênero dos entrevistadores e mediadores de cada pesquisa. Reflete-se que há necessidade da reflexividade dos pesquisadores sobre suas posições, escolha do tema, critérios de seleção dos participantes, papel do mediador para avaliar as razões das dificuldades no recrutamento dos participantes e impactos nos resultados das pesquisas em área rural. Conclusão: o processo de recrutamento dos participantes deve ser melhor considerado pelos pesquisadores para a maior qualidade das pesquisas qualitativas.

DESCRITORES: Pesquisa qualitativa. População rural. Família. Sujeitos da pesquisa. Enfermagem.

\section{PROCESS OF SEARCHING FAMILIES FOR DATA COLLECTION IN QUALITATIVE RESEARCH}

\begin{abstract}
Objective: to reflect on the process of searching families in the rural area to participate in research.

Method: reflection on the search process undertaken in two studies (A and B) based on four factors: theme, selection criteria, role of the mediator and researcher's position concerning gender, age, profession and affiliation.

Results: the analysis of these four factors in the search process for the participants shows that the mediators' help was fundamental to select and get access to the families at their homes. Difficulty was observed to hear different family members. In study A, adult men were the main participants and, in study B, adult women, in accordance with the interviewers and mediators' gender in each research. The researchers need to reflect on their positions, choice of the theme, participant selection criteria, role of the mediator to assess the difficulties in recruiting the participants and impacts in the results of research in the rural area. Conclusion: the researchers should better consider the recruitment process of the participants in order to enhance the quality of qualitative research.
\end{abstract}

DESCRIPTORS: Qualitative research. Rural population. Family. Research subjects. Nursing. 


\section{PROCESO DE BÚSQUEDA DE FAMILIAS PARA LA RECOLECCIÓN DE DATOS EN LA INVESTIGACIÓN CUALITATIVA}

\section{RESUMEN}

Objetivo: reflexionar sobre el proceso de búsqueda de familias para la investigación en el área rural.

Método: reflexión sobre el proceso de búsqueda realizado en dos investigaciones (A y B) a partir de cuatro factores: el tema, los criterios de selección, el papel del mediador y la posición del investigador en relación al género, edad, profesión y filiación.

Resultados: el análisis de estos cuatro factores en el proceso de búsqueda de los participantes muestra que el auxilio de los mediadores fue fundamental para seleccionar y tener acceso a las familias en sus domicilios. Hubo dificultad para oír a los diferentes miembros de las mismas. En la investigación A hubo una mayor participación de los hombres adultos, y en la investigación B de las mujeres adultas, lo que coincidió con el género de los entrevistadores y mediadores de cada investigación. Existe la necesidad de reflexivilidad de los investigadores sobre sus posiciones, la elección del tema, los criterios de selección de los participantes, la función del mediador para evaluar las razones de las dificultades en el reclutamiento de los participantes y los impactos en los resultados de las investigaciones en el área rural.

Conclusión: el proceso de reclutamiento de los participantes debe ser mejor considerado por los investigadores para una mejor cualidad de las investigaciones cualitativas.

DESCRIPTORES: Investigación cualitativa. Población rural. Familia. Sujetos de la investigación. Enfermería.

\section{INTRODUÇÃO}

Apesar dos avanços recentes, a pesquisa qualitativa ainda vem sendo aperfeiçoada e os pesquisadores desta modalidade lutam para ganhar cada vez mais credibilidade no mundo científico. A literatura ${ }^{1}$ alerta que o planejamento e a execução da pesquisa qualitativa, principalmente a seleção e a busca dos participantes, traduzem-se em certo envolvimento emocional, exigem o planejamento de tempo e a capacidade de estabelecer relações que sejam frutíferas para alcançar resultados significativos, do ponto de vista dos projetos de pesquisa. Na pesquisa qualitativa que tem como participantes grupos familiares, tais aspectos adquirem capital importância, sobretudo em seus domicílios, por possibilitar, além de entrevistas, outras técnicas como a observação. Isto se torna particularmente mais difícil quando se buscam famílias em áreas remotas da zona rural ou mesmo em áreas urbanas de difícil acesso.

No que se refere ao processo de seleção e de busca dos participantes em estudos qualitativos, a ênfase é posta sobre os critérios de seleção dos participantes e estratégias de coleta de dados. No entanto, o processo da busca (recrutamento) dos participantes pesquisados geralmente é subestimado. A falta de foco sobre esta etapa esconde uma parte importante da estratégia de coleta de dados nas pesquisas qualitativas, particularmente nas entrevistas. $^{2}$

O processo de recrutamento contribui diretamente para a qualidade da pesquisa, haja vista que os pesquisadores buscam as oportunidades que se oferecem no contexto do estudo para o seu desenvolvimento e estruturam o material final em uma interação com os critérios pré-definidos para eleger os participantes e os mediadores. ${ }^{2}$ Mediador é uma pessoa que, por sua posição formal ou informal e relações sociais, pode facilitar o contato entre pesquisador e os potenciais informantes. ${ }^{3}$

Ainda se enfatiza que a falta de foco no recrutamento compromete a reflexividade que tem se tornado importante na pesquisa qualitativa nas últimas décadas. ${ }^{2} \mathrm{~A}$ reflexividade é vista como um processo contínuo de diálogo interno e autoavaliação crítica da posição do pesquisador, bem como um reconhecimento ativo e explícito de sua posição, que pode afetar o processo e os resultados da pesquisa. ${ }^{4-5}$

São quatro fatores a serem considerados para buscar os participantes2: 1) significados e efeitos do tópico a ser pesquisado; 2) significados e efeitos da amostra, dos critérios de inclusão e exclusão; 3 ) significados e efeitos do mediador (gatekeeper) 3; e 4) significados e efeitos da posição e situação do pesquisador (capacidade de reflexão sobre a posição em termos de gênero, idade, origem, etnicidade). ${ }^{4}$

No que tange à pesquisa qualitativa com famílias, deve-se mencionar que há controvérsias acerca do que realmente são pesquisas com famílias ou pesquisas relacionadas a famílias. Os quatro focos de interesse neste tipo de pesquisa são: a) o indivíduo, como membro da família, em que a família serve como contexto e a ênfase está no fenômeno (sujeito) individual; b) o indivíduo como parte de um subgrupo familiar, no qual tanto indivíduos como relacionamentos são estudados, tendo a família como contexto; c) famílias como um grupo, que tem a família como foco; e d) indivíduos e famílias, em que o foco pode ser dois ou mais níveis do subsistema, o qual inclui indivíduos, relacionamentos e famílias. ${ }^{6}$ A pesquisa que utiliza as respostas individuais de membros da família é denominada pesquisa relativa à família. Assim, a questão do acesso às famílias tem implicação direta com o foco 
de interesse delineado pela pesquisa e as formas de aproximação com o fenômeno. ${ }^{6}$

Buscar famílias na área rural brasileira também se constitui um desafio importante para os pesquisadores, dada a realidade encontrada nas diferentes regiões. Mesmo no Sul do Brasil, ainda se encontram municípios de difícil acesso rodoviário e meios de comunicação em geral. Isto dificulta a utilização de estratégias atuais na busca de dados, tais como a internet, ou dificulta até mesmo um simples contato telefônico para marcar uma entrevista. A dificuldade de acesso e o contato com áreas rurais, muitas vezes, representam por si só uma importante restrição para conhecer melhor esta realidade, privilegiando as pesquisas em centros urbanos.

A partir do processo de busca de famílias para pesquisa na área rural, em duas pesquisas realizadas com famílias, em ciências da saúde e ciências sociais aplicadas no campo da enfermagem e da administração, respectivamente, o presente artigo teve por objetivo refletir o processo de busca de famílias para pesquisa na área rural em dois municípios catarinenses.

Além de ampliar a produção do conhecimento sobre a pesquisa qualitativa, acredita-se que essa reflexão tem potencial para contribuir com os pesquisadores que utilizam tal modalidade de pesquisa, em especial no que concerne ao processo de recrutamento dos participantes dos estudos.

\section{Identificação e o tema das duas pesquisas}

O tema de uma pesquisa tem um significativo impacto sobre a seleção dos participantes pesquisados e, desta forma, na delimitação das experiências e processos de significação, que são mais susceptíveis de serem incluídos ou não no objeto da análise do pesquisador. O tema da pesquisa, portanto, pode delimitar facilidades e também dificuldades para se obter os dados de uma pesquisa. ${ }^{2}$

\section{Pesquisa A}

Constituiu-se em uma tese de doutorado de um Programa de Pós-Graduação em Administração, cujo objetivo foi compreender a orientação da ação nos modos de produção entre famílias ligadas à atividade agrícola na pequena propriedade rural. Teve como principal referencial teórico a dimensão epistemológica de base interpretativista, a partir das dimensões de análise da teoria da ação social weberiana. ${ }^{7} \mathrm{O}$ local da pesquisa se situou entre duas localidades com predominância de agricultores de ascendência alemã na área rural, no interior do município de São Bonifácio, Santa Catarina, Brasil. O desenho adotado foi a pesquisa qualitativa, estudo de múltiplos casos, compreendendo ao todo 13 famílias, tendo a participação total de 27 indíviduos nas entrevistas. As primeiras famílias foram indicadas por um mediador, posteriormente por indicação das famílias entrevistadas. Foram realizadas entrevistas e observação não participante. ${ }^{8}$

\section{Pesquisa B}

Trata-se também de uma tese, de um Programa de Pós-Graduação em Enfermagem, sendo que o objetivo foi compreender como são interpretadas as rotinas e os rituais de cuidado para a promoção da saúde, nas famílias rurais em transição inesperada de situações de pós-desastre. O referencial teórico analítico foi o interpretativismo, ${ }^{9}$ sustentando-se também no referencial teórico das rotinas e dos rituais de cuidado ${ }^{10-11}$ e na teoria do ciclo vital familiar. A pesquisa foi realizada em uma localidade rural de um município do Vale do Itajaí/SC, atingida por um desastre ambiental climático de grandes proporções, ocorrido em 2008. A abordagem do estudo foi qualitativa, estudo de caso múltiplo, composto por seis famílias, totalizando doze indivíduos adultos entrevistados, dez crianças entre 0 e 12 anos e três adolescentes. As famílias foram inicialmente indicadas pelos mediadores e posteriormente por outras famílias. A observação participante foi gradativa e se deu concomitante à realização de entrevistas narrativas no domicílio de cada família, após um ano do desastre. ${ }^{10-11}$

\section{Significados dos critérios de seleção na busca das famílias}

Para o critério de seleção dos participantes em uma pesquisa qualitativa, "uma amostra qualitativa, melhor denominada seleção de casos, o olhar do pesquisador é menos fixado de antemão e se desloca incessantemente de um ponto ao outro" ${ }^{12: 1}$ Isto significa que o processo de busca dos participantes da pesquisa é orientado para o alcance dos objetivos e simultaneamente é um trabalho exploratório. $\mathrm{O}$ processo de seleção não é um processo cientificamente neutro, mas imbuído de decisões subjetivas. A busca dos participantes se constitui em um processo, no qual é possível dar mais voz a alguns entrevistados e silenciar outros. ${ }^{2}$

Na pesquisa A, os critérios de inclusão propostos foram: famílias de agricultores detentores de pequenas propriedades rurais e que apresentavam alguma atividade de produção agrícola na qual o 
trabalho realizado fosse compartilhado entre os integrantes deste grupo familiar. Consideraram-se os diferentes tipos de famílias nucleares e extensas, residindo ou não no mesmo domicílio ou propriedade, desde que contemplassem o critério do trabalho agrícola compartilhado. A análise dos dados concomitante à coleta de dados mostrou diferentes modos de produção agrícola, o que levou à busca de mais famílias que contemplassem modos de produção similares e ou diferenciados.

Na pesquisa B, considerou-se a família nuclear formada por pais ou um dos pais e filhos. Quanto aos critérios de inclusão, inicialmente a pesquisadora buscou famílias com no mínimo uma criança com idade entre 0 e 12 anos, o que caracterizaria famílias na fase do ciclo vital de aquisição. Quando solicitada a ajuda aos membros da equipe de saúde da localidade em questão, para a seleção de famílias que porventura pudessem participar da pesquisa, deu-se maior ênfase ao critério da presença de crianças. Porém, após integração ao campo, constatou-se a dificuldade em buscar famílias nucleares somente com crianças, visto que as famílias indicadas, além de terem crianças, também eram compostas de filhos na adolescência, caracterizando as famílias na fase adolescente do ciclo vital. Assim, foi descartada a delimitação de casos a partir de uma fase específica do ciclo vital familiar, e considerou-se a realidade local e a condição de acessibilidade às famílias.

\section{O papel dos mediadores na busca das famílias}

Uma estratégia de busca dos informantes na pesquisa qualitativa envolve um mediador também denomindado de porteiro da pesquisa. ${ }^{3}$ Os mediadores influenciam diretamente na busca dos informantes e, consequentemente, no material produzido para a pesquisa, dada sua familiaridade com o contexto. Sua influência pode também limitar o âmbito da pesquisa, de modo que o pesquisador deve estar atento ao eventual direcionamento que porventura o mediador possa ter..$^{2-3}$ Ademais, dada a sua familiaridade com o universo pesquisado, sua relevância potencializa o processo de seleção de casos. Deste modo, relata-se como foi o processo de identificação dos mediadores nas duas pesquisas, sua atuação e como foram orientados.

Pesquisa A: a entrada no campo foi gradativa desde a fase da concepção do projeto. Em função da distância do domicílio do pesquisador com o local, havia a necessidade de hospedagem e permanência no campo por alguns dias, incluindo finais de semana em cada etapa da pesquisa. A hospedagem foi na casa de um familiar do pesquisador que re- side na parte central da localidade. Isto permitiu o estabelecimento de contatos com famílias das duas localidades, a observação, contatos e visitas preliminares a potenciais famílias-alvo da pesquisa. Na fase da coleta de dados, a falta de telefones disponíveis entre as famílias nas duas localidades da pesquisa e a distância entre as suas respectivas propriedades dificultavam fazer contatos prévios. Assim, era preciso uma visita anterior à entrevista, para agendamento, que era feita para o início da tarde do mesmo dia ou para o dia seguinte. Em virtude da disponibilidade do pesquisador e das famílias, diversas entrevistas foram realizadas em períodos de finais de semana.

Este processo foi facilitado por um mediador, pessoa do círculo familiar do próprio pesquisador, nascido no município, dono de uma propriedade rural na localidade, e que mantém estreitas relações com moradores. O mediador teve conhecimento do objeto da pesquisa, inicialmente através de conversas informais, e posteriormente lhe foi disponibilizada a leitura do projeto. O papel do mediador que acompanhava as visitas foi fundamental, para estabelecer um clima de confiança entre o pesquisador e os pesquisados. Principalmente no início de cada entrevista, enquanto o pesquisador ajustava o gravador e buscava organizar o roteiro de questionamentos, o facilitador conduzia uma conversa informal com os entrevistados, facilitando o trabalho do pesquisador. Em alguns casos, durante as observações da propriedade e mesmo em algumas entrevistas, o mediador auxiliava a fazer novos questionamentos sobre aqueles assuntos que careciam de mais detalhes. Em outras entrevistas, o facilitador se entretinha com outras pessoas da família, enquanto as perguntas eram dirigidas para um membro específico. Após as visitas, as conversas com o mediador possibilitaram reflexões úteis para o amadurecimento da análise dos dados.

Pesquisa B: dada a trajetória profissional da pesquisadora, a entrada no campo ocorreu através da Unidade Básica de Saúde (UBS). O primeiro contato e a recepção da pesquisadora na unidade foram feitos por um enfermeiro já conhecido que apresentou a UBS, assim como a localidade pela qual a equipe de saúde era responsável. Depois de apresentada a proposta de pesquisa para a equipe de saúde e informados os critérios de seleção, a pesquisadora contou com o relato, em especial, do enfermeiro e das agentes comunitárias de saúde a respeito das situações de saúde das famílias que eles visitavam. Também forneceram uma versão prévia sobre o histórico de cada família no desas- 
tre, que indicavam o potencial de cada uma como "caso interessante" para a participação na pesquisa. Estas agentes comunitárias tornaram-se, por consequência, as mediadoras na pesquisa. Contudo, tal forma de comunicar o "estado geral" das famílias expressava, por um lado, o que os colaboradores entenderam como seus critérios de escolha de famílias, que eram aquelas consideradas mais problemáticas. Em outro sentido, isto pode significar também uma espécie de interesse da equipe de saúde na atuação da pesquisadora, que é uma profissional da saúde.

No primeiro contato com as famílias, as agentes comunitárias de saúde faziam a aproximação da pesquisadora, apresentando-a como "uma enfermeira interessada em conversar sobre uma pesquisa". As primeiras visitas ocorreram tanto em dias úteis da semana, como nos finais de semana, o que possibilitou também a observação sobre o que acontece na comunidade nesses diferentes momentos. Permitiu ainda detectar as famílias que só poderiam ser contatadas nos finais de semana.

\section{Sobre a posição do pesquisador na busca dos entrevistados}

A reflexividade consiste na maior estratégia de controle de qualidade na pesquisa qualitativa. ${ }^{4-5}$ $\mathrm{O}$ entendimento de como as experiências vividas e as características do pesquisador, tais como gênero, experiências pessoais e profissionais, filiação, tem efeitos significativos nas pesquisas podem impactar e, portanto, são de suma importância.

Na pesquisa A, sobre a posição de gênero, a maior parte das entrevistas se deu junto aos homens das famílias, com algumas exceções. Reflete-se que dadas as situações do acesso, que era feito na primeira visita, havia uma tendência de os homens receberem e mostrarem a propriedade ao pesquisador e mediador, que eram ambos do sexo masculino. Nas entrevistas, os homens faziam questão de responder pelas questões da propriedade e mesmo naquelas em que houve a participação de mulheres (a maioria esposas), os homens exerciam certa liderança.

Quanto às experiências pessoais e profissionais, o pesquisador já tinha um envolvimento desde a infância com as duas localidades. Assim, mesmo sendo da área urbana, o pesquisador possuía identificação e vivência rural local, além da mesma origem étnica das famílias. A posição profissional do pesquisador não teve relevância na busca das famílias, ao contrário do que ocorreu na pesquisa B.

Na pesquisa $B$, em relação ao gênero, a pesquisadora e as mediadoras eram do sexo feminino, assim, todas as tratativas prévias e a recepção no domicílio eram feitas pelas mulheres. No tocante à posição profissional, inicialmente o papel da enfermeira se mostrava predominante, e as famílias participantes se sentiram no direito de pedir opiniões acerca das questões de saúde na família. Acredita-se que isto tenha contribuído sobremaneira para a relação de confiança, sobressaindo-se gradativamente o papel de pesquisadora, sem, no entanto, se desvencilhar do papel da enfermeira. A filiação da pesquisadora, neta de famílias de agricultores e residentes na região, teve que ser resgatada nos momentos em que, consciente de sua condição urbana, buscava empatia sobre temas próprios da vida rural.

\section{Refletindo a respeito do processo de busca dos participantes}

Como já mencionado anteriormente, quem faz pesquisa qualitativa ainda precisa lutar para garantir a sua legitimidade, desse modo, o esforço dos pesquisadores precisa se voltar para a garantia da sua qualidade. Tradicionalmente, na formação do pesquisador, discutem-se as diferenças entre pesquisa qualitativa e pesquisa quantitativa, e uma das primeiras lições apreendidas e reiteradas pela bibliografia clássica desta área é a de que o pesquisador é o maior instrumento da pesquisa qualitativa. Sendo assim, a busca da qualidade está ligada aos atos de reflexão sobre o processo da pesquisa. ${ }^{5}$

Os estudos qualitativos tratam dos fenômenos das experiências humanas e não das populações, cuja amostra é escolhida aleatoriamente. Assim, para a seleção dos participantes, o interesse está na subjetividade deles e, sobretudo, na experiência que eles têm com relação ao tema. ${ }^{5}$ Desta maneira, no processo de recrutamento, o problema principal não se resume em achar os participantes para uma pesquisa,o desafio está em achar o participante certo. ${ }^{1}$

Na questão da pesquisa com famílias, ${ }^{6}$ principalmente na qual se buscam famílias como um grupo, este desafio se torna ainda maior. Além de achar as famílias em que diferentes membros possam e queiram compartilhar suas experiências, o pesquisador também precisa ganhar acesso e estabelecer interações que resultem em dados frutíferos para os objetivos do estudo. ${ }^{5}$

Na apresentação feita com exemplos das duas pesquisas com famílias na área rural, os significados e efeitos do tema, os critérios de inclusão e exclusão; o mediador; a reflexividade sobre a posição e situação do pesquisador (capacidade de reflexão relativa à posição em termos de gênero, idade, origem, etnici- 
dade $)^{4}$ possibilitaram fazer algumas reflexões sobre a busca para a participação em pesquisas.

As entrevistas dos diferentes membros das famílias, bem como a observação das propriedades, foram de fundamental importância para a obtenção dos dados nas duas pesquisas, visando atingir os objetivos dos estudos e estabelecer certo vínculo de interação, respeito e "confiança para o com a proposta da pesquisa" que oportunizassem as idas e vindas do trabalho de campo do pesquisador ao longo do período de imersão na coleta dos dados. Neste sentido, ganhar acesso a tais famílias na área rural no processo de busca teve inúmeros desafios.

Acerca do tema a ser pesquisado, buscar famílias para falar a respeito dos modos de produção da atividade agrícola na pesquisa A e falar das rotinas de cuidado na promoção de saúde um ano pós-desastre na pesquisa $B$, o desafio foi menor. Dependendo do tema, a entrevista é uma oportunidade positiva para o entrevistado expor as suas experiências. ${ }^{2}$ No entanto, tratando-se de pesquisa com famílias, a dificuldade maior foi ouvir diferentes membros delas. Os jovens e adolescentes apresentavam maiores restrições de falar com os pesquisadores, ou, quando falavam, demonstravam menos interesse.

Dessa forma, determinados temas podem ser difíceis de serem abordados, tanto para os entrevistados como pelos entrevistadores. ${ }^{2} \mathrm{Ou}$, ainda, temas que entrevistados não têm interesse. Portanto, alguns são discutidos com maior frequência nas pesquisas, resultando em mais conhecimento produzido quanto a esses em detrimento de outros. Nas pesquisas com famílias, certos temas direcionam para a participação de um porta-voz da família, podendo se obter apenas dados parciais relativos ao tema. A subjetividade dos entrevistados que compõem as famílias pesquisadas garante que um mesmo tema seja percebido, interpretado e discutido sob diferentes pontos de vista nas entrevistas, o que, qualitativamente, se torna interessante para a análise dos assuntos pesquisados.

Descrever a respeito da dificuldade que os diferentes membros de uma família, ou demais participantes, têm para falar sobre determinados tópicos de pesquisa poderá auxiliar outros pesquisadores a refinar suas estratégias de busca e desenvolver estratégias de interação capazes de obter dados.

Com relação aos significados e efeitos dos critérios de inclusão e exclusão, na busca das famílias, em ambas as pesquisas, para o recrutamento, os participantes sofreram alterações no transcorrer do estudo. No exemplo da pesquisa B, a pesquisadora, depois de ambientada no campo, descobriu que não poderia se limitar a famílias somente com crianças, avançando para incluir também aquelas com filhos adolescentes. Isto corrobora o que aponta a literatura ${ }^{2}$ de que os critérios para a seleção dos participantes se constituem em um trabalho exploratório para o alcance dos objetivos da pesquisa. Quando os critérios são excessivamente fechados, reproduzem ideias culturais sobre quem pode responder a determinados temas, sem explorar realmente $o$ tema com diferentes pessoas, mesmo as de mais difícil acesso. Isto leva novamente ao argumento de que certos grupos são mais estudados que outros. E nas pesquisas com famílias, alguns integrantes das famílias são mais facilmente acessados que outros, implicando diretamente no conhecimento produzido.

Logo, os pesquisadores devem descrever nos seus relatórios, além das razões dos critérios de inclusão e exclusão, as escolhas quanto ao espaço, tempo e as estratégias de recrutamento. Devem discutir abertamente as dificuldades encontradas na seleção de determinados grupos de pessoas, famílias, integrantes de famílias, utilizando a reflexividade, descobrindo assim tabus e pré-conceitos relacionados tanto ao pesquisador como ao pesquisado. ${ }^{2}$

Muitos desafios foram vencidos na busca das famílias na área rural, no exemplo das duas pesquisas, em grande parte, pela maneira como ocorreu a entrada no campo com o auxílio dos mediadores. Os dados das pesquisas apontam que, na pesquisa A, a entrada se deu através de um mediador da própria família do pesquisador. $\mathrm{Na}$ pesquisa $\mathrm{B}, \mathrm{o}$ acesso aconteceu por meio do elo profissional com agentes de saúde, o que determinou a escolha dos mediadores e as experiências que se sucederam, influenciando tanto na seleção e acesso das famílias, quanto na produção dos dados. A influência dos mediadores na busca das famílias sobressaiu especialmente na pesquisa $B$, indicando a necessidade do pesquisador de se certificar da real compreensão dos objetivos da pesquisa pelos mediadores, uma vez que as famílias indicadas eram somente as que precisavam de assistência, na visão dos agentes de saúde, e não famílias em geral que passaram pela transição de um desastre. Isto vem ao encontro da literatura, ${ }^{2}$ no sentido de atentar para a escolha do mediador, que pode ser um facilitador, mas pode também silenciar vozes importantes na produção dos dados e no resultado final da pesquisa. Ainda, há de se considerar, durante a pesquisa, qual é o poder do pesquisador e do mediador durante o processo do recrutamento. ${ }^{3}$ 
Assim, dada a importância do mediador em todo o processo de pesquisa tanto para o sucesso como para o fracasso, os pesquisadores devem estabelecer relações de confiança, esclarecer os objetivos da pesquisa e os critérios de seleção ao mediador. Sugere-se que o pesquisador solicite a descrição subjetiva do mediador sobre as razões de indicação dos participantes para a pesquisa. ${ }^{2}$ Nos relatos da pesquisa, devem estar descritos a razão da escolha dos mediadores, a relação entre o pesquisador, a orientação recebida anterior à pesquisa e o papel do mediador em todo o processo da pesquisa.

Em relação à posição do pesquisador na busca e na motivação para recrutar as famílias e seus integrantes, o exemplo das duas pesquisas mostra o impacto que tiveram o gênero e as experiências prévias dos pesquisadores e também dos mediadores.

No que concerne à busca dos entrevistados dentro da família, tanto na pesquisa A, visando compreender os modos de produção agrícola familiar, como na pesquisa B, de compreender as rotinas de cuidado da família no pós-desastre, tinha-se como desafio obter dados de diferentes integrantes das famílias. Na reflexão acerca da posição dos pesquisadores e dos mediadores nesta busca, foi de que a questão de gênero, aliada ao tema da pesquisa e à trajetória familiar, proporcionaram experiências diferentes nas duas pesquisas. $\mathrm{Na}$ pesquisa $\mathrm{A}$, o pesquisador e o mediador eram do sexo masculino, sendo que houve maior participação dos homens adultos, as mulheres tiveram participação menor e jovens participaram com maior dificuldade. Na pesquisa $B$, a pesquisadora e as mediadoras eram do sexo feminino, a participação predominante foi feminina, os jovens também tiveram menor participação.

Pode-se inferir aqui que tiveram grande relevância os valores relacionados aos papéis que cada um assume na família: as atividades agrícolas são assunto de homem, as rotinas de cuidado estão mais afetas às mulheres. Os jovens solteiros ou adolescentes demonstraram menos interesse pelos dois temas, talvez influenciados pelo gênero e pela idade dos pesquisadores. A esse respeito, tem-se o exemplo de uma pesquisa ${ }^{2}$ com o objetivo de descobrir sobre o papel social das crianças, em que a pesquisadora era mulher e mãe de classe média, os critérios de inclusão eram homens e mulheres de todas as classes. Com muita facilidade houve o recrutamento das mulheres da classe media, sendo que não foi possível recrutar homens da classe trabalhadora. Os autores mencionaram que a classe, o gênero da pesquisadora e as ideias preconcebidas de igualdade de gênero sobre o tema tiveram impacto no recrutamento.

A posição profissional do pesquisador foi mais relevante na pesquisa $B$, e teve implicações na busca das famílias, como já destacado anteriormente, relacionado com o entendimento das mediadoras acerca dos objetivos da pesquisa e do papel do pesquisador. Quanto à posição dos pesquisadores na aproximação com a área rural, a filiação do pesquisador, na pesquisa A, foi relevante, na pesquisa $B$, em algumas situações, também auxiliou na aproximação com as famílias.

Por último, esses dados nos remetem novamente ao exercício da reflexividade como uma estratégia de controle da qualidade em todo o processo da pesquisa..$^{4-5} \mathrm{E}$ a importância de se exercer o controle constante para compreender os fatores que motivam ou não as pessoas, famílias, grupos a participarem de pesquisas.

Os grupos de pesquisa deveriam trazer à luz e discutir mais sobre os motivos pelos quais alguns temas são mais pesquisados em certos grupos de pessoas. As respostas para tais perguntas, os pesquisadores podem buscar na análise mais detalhada do processo de busca dos participantes. ${ }^{2}$ Por que existem resistências? Esta fase da busca/recrutamento tem muitos desafios a serem enfrentados e, muitas vezes, são difíceis de serem antecipados, tendo consequências sobre os resultados da pesquisa.

\section{CONCLUSÃO}

A busca pela qualidade da pesquisa, aqui em especial na modalidade qualitativa, requer atenção dos pesquisadores em todo o seu processo, da concepção à sua conclusão. O método, considerado como cérebro da pesquisa, precisa necessariamente ser bem delineado para não comprometer a qualidade do estudo e, deste modo, não se deve subestimar a importância do processo de recrutamento dos participantes.

Nessa reflexão destacou-se o quanto o tema da pesquisa, os critérios de seleção, o papel do mediador e a posição do pesquisador no recrutamento dos participantes podem ser um facilitador na investigação, a depender do modo como foram pensados e executados.

Portanto, aponta para a necessidade de que a descrição do processo de busca dos participantes deve ser melhor considerada pelos pesquisadores para uma maior credibilidade e qualidade das pesquisas qualitativas. Daí a importância do presente estudo, além do seu ineditismo nessa área do conhecimento. 


\section{REFERÊNCIAS}

1. Wigfall VG, Brannen J, Mooney A, Parutis V. Finding the right man: recruiting fathers ininter-generational families across ethnic groups. Qualitative Res [Internet]. 2012 [cited 2016 Dec 07]; 13(5):591-607. Available from: http://dx.doi.org/10.1177/1468794112446109

2. Kristensen GK, Ravn MN. The voices heard and the voices silenced: recruitment processes in qualitative interview studies. Qualitative Res [Internet]. 2015 [cited 2016 Dec 07]; 15(6):722-37. Available from: http://dx.doi.org/10.1177/1468794114567496

3. Lund R, Panda SM, Dhal MP. Narrating spaces of inclusion and exclusion in research collaboration - researcher-gatekeeper dialogue. Qualitative Res [Internet]. 2016 [cited 2016 Dec 07]; 16(3):280-92. Available from: http://dx.doi. org/10.1177/1468794115611208

4. Berger R. Now I see it, now I don't: researcher's position and reflexivity in qualitative research. Qualitative Res [Internet]. 2015 [cited 2016 Dec 07]; 15(2):219-34. Available from: http://dx.doi. org/10.1177/1468794112468475

5. Benjumea CC. The quality of qualitative research: from evaluation to attainment. Texto Contexto Enferm [Internet]. 2015 [cited 2016 Dec 07], 24(3):883-90. Available from: http://dx.doi.org/10.1590/0104070720150001150015

6. Ângelo M, Bousso RS, Rossato LM, Damião EBC, Silveira AO, Castilho AMCM, et al. Família como categoria de análise e campo de investigação em enfermagem. Rev Esc Enferm USP [Internet]. 2009 Dec [cited 2016 Dec 07]; 43(spe2):1337-41. Available from: http://dx.doi.org/10.1590/S008062342009000600033.

7. Weber M. Economia e Sociedade: fundamentos da sociologia compreensiva. 4 ed. 2 v. Brasilia (DF): Ed. UNB; 2009.

8. Boehs CGE. A orientação da ação nos modos de organizar a produção agrícola entre as famílias de agricultores no município de São Bonifácio-SC [tese]. Curitiba (PR): Programa de Mestrado e Doutorado em Administração, Universidade Positivo; 2016.

9. Geertz C. A interpretação das culturas. Rio de Janeiro (RJ): LTC; 1989.

10. Fernandes GCM, Boehs AE, Heidemann ITSB. Social support during the family transition following a natural disaster. Texto Contexto Enferm [Internet]. 2013 [cited 2016 Dec 07], 22(4):1098-105. Available from: http://dx.doi.org/10.1590/S0104-07072013000400028

11. Fernandes GCM. Rotinas e rituais de cuidado nas famílias rurais em transição inesperada do pósdesastre [tese]. Florianópolis (SC): Programa de PósGraduação em Enfermagem, Universidade Federal de Santa Catarina; 2011.

12. Pires PA. Amostragem e pesquisa qualitativa: ensaio teórico e metodológico. In: Poupart J, Deslauriers JP, Groulx L, Laperrére A, Mayer R, Pires AP. A pesquisa qualitativa: enfoques epistemológicos e metodológicos. Petrópolis (RJ): Ed. Vozes, 2008, p.154-211.
Correspondência: Astrid Eggert Boehs

Rua: Valter De Bonna Castelan 429

88037-300 - Jardim Anchieta, Florianópolis, SC, Brasil

E-mail: astridboehs@hotmail.com
Recebido: 09 de fevereiro de 2017

Aprovado: 27 de julho de 2017

This is an Open Access article distributed under the terms of the Creative Commons (CC BY). 